Journal of Applied AnALysis

Vol. 13, No. 1 (2007), pp. 1-11

\title{
ASYMPTOTIC BEHAVIOR OF INEXACT ORBITS FOR A CLASS OF OPERATORS IN COMPLETE METRIC SPACES
}

\author{
D. BUTNARIU, S. REICH, and A. J. ZASLAVSKI \\ Received September 19, 2005 and, in revised form, June 1, 2006
}

\begin{abstract}
We exhibit a class of nonlinear operators with the property that their iterates converge to their unique fixed points even when computational errors are present. We also show that most (in the sense of the Baire category) elements in an appropriate complete metric space of operators do, in fact, possess this property.
\end{abstract}

\section{INTRODUCTION}

Assume that $(X, \rho)$ is a complete metric space and let the operator $A: X \rightarrow X$ have the following properties:

(A1) there exists a unique $x_{A} \in X$ such that $A x_{A}=x_{A}$;

(A2) $A^{n} x \rightarrow x_{A}$ as $n \rightarrow \infty$, uniformly on all bounded subsets of $X$;

(A3) $A$ is uniformly continuous on bounded subsets of $X$;

(A4) $A$ is bounded on bounded subsets of $X$.

2000 Mathematics Subject Classification. Primary: 47H10, 54E50, $54 \mathrm{E} 52$.

Key words and phrases. Complete metric space, generic property, inexact orbit, iteration.

ISSN 1425-6908 C Heldermann Verlag. 
Many operators with these properties can be found, for example, in [1], [2], [4], [7], [8], [9], [11]-[13]. We mention, in particular, the classes of operators introduced by Rakotch [9] and Browder [1]. Note that if $X$ is either a closed, convex subset of a Banach space or a closed, $\rho$-convex subset of a complete hyperbolic metric space [10], then (A4) follows from (A3).

In view of (A2), it is natural to ask if the convergence of the orbits of $A$ will be preserved even in the presence of computational errors. In this paper we provide affirmative answers to this question. More precisely, we have the following results.

Theorem 1.1. Let $K$ be a nonempty, bounded subset of $X$ and $\varepsilon>0$. Then there exist $\delta=\delta(\varepsilon, K)>0$ and a natural number $N$ such that for each natural number $n \geq N$, and each sequence $\left\{x_{i}\right\}_{i=0}^{n} \subset X$ which satisfies

$$
x_{0} \in K \text { and } \rho\left(A x_{i}, x_{i+1}\right) \leq \delta, i=0, \ldots, n-1,
$$

the following inequality holds:

$$
\rho\left(x_{i}, x_{A}\right) \leq \varepsilon, i=N, \ldots, n .
$$

Corollary 1.1. Assume that $\left\{x_{i}\right\}_{i=0}^{\infty} \subset X,\left\{x_{i}\right\}_{i=0}^{\infty}$ is bounded, and that

$$
\lim _{i \rightarrow \infty} \rho\left(A x_{i}, x_{i+1}\right)=0 \text {. }
$$

Then $\rho\left(x_{i}, x_{A}\right) \rightarrow 0$ as $i \rightarrow \infty$.

Theorem 1.2. Let $\varepsilon>0$. Then there exists $\delta=\delta(\varepsilon)>0$ such that for each sequence $\left\{x_{i}\right\}_{i=0}^{\infty} \subset X$ which satisfies

$$
\rho\left(x_{0}, x_{A}\right) \leq \delta \text { and } \rho\left(x_{i+1}, A x_{i}\right) \leq \delta, i=0,1, \ldots,
$$

the following inequality holds:

$$
\rho\left(x_{i}, x_{A}\right) \leq \varepsilon, i=0,1, \ldots
$$

These results show that, roughly speaking, in order to achieve an $\varepsilon$ approximation of $x_{A}$, it suffices to compute inexact orbits of $A$, that is, sequences $\left\{x_{i}\right\}_{i=0}^{\infty}$ such that

$$
x_{0} \in X \text { and } \rho\left(x_{i+1}, A x_{i}\right) \leq \delta \text { for any } i \geq 0,
$$

where $\delta$ is a sufficiently small positive number.

However, sometimes the operator $A$ is not given explicitly and only some approximation of it, $B_{i}$, is available at each step $i$ of the inexact orbit computing procedure. The next result shows that for certain operators $A$, the procedure of approximating $x_{A}$ by inexact orbits is stable in the sense that, even in this case, the orbits determined by the sequence of operators $B_{i}$ approach $x_{A}$ provided that each $B_{i}$ is a sufficiently accurate approximation 
of $A$ in the topology of uniform convergence on bounded subsets of $X$. To be precise, we set, for each $x \in X$ and $E \subset X$,

$$
\rho(x, E)=\inf \{\rho(x, y): y \in E\} .
$$

Denote by $\mathcal{A}$ the set of all self-mappings $A: X \rightarrow X$ which have properties (A3) and (A4). Fix $\theta \in X$. For each natural number $n$, set

$$
E_{n}=\{(A, B) \in \mathcal{A} \times \mathcal{A}: \rho(A x, B x) \leq 1 / n \text { for all } x \in B(\theta, n)\} .
$$

We equip the set $\mathcal{A}$ with the uniformity determined by the base $E_{n}, n=$ $1,2, \ldots$ This uniformity is metrizable by a complete metric.

Denote by $\mathcal{A}_{\text {reg }}$ the set of all mappings $A \in \mathcal{A}$ which satisfy (A1) and (A2), and by $\overline{\mathcal{A}}_{\text {reg }}$ the closure of $\mathcal{A}_{\text {reg }}$ in $\mathcal{A}$.

Theorem 1.3. Assume that $A \in \mathcal{A}_{\mathrm{reg}}$ and $x_{A}$ is the fixed point of $A$. Let $m, \varepsilon>0$. Then there exist a neighborhood $\mathcal{U}$ of $A$ in $\mathcal{A}$ and a natural number $N$ such that for each $x \in B(\theta, m)$, each integer $n \geq N$, and each sequence $\left\{B_{i}\right\}_{i=1}^{n} \subset \mathcal{U}$,

$$
\rho\left(B_{i} \ldots B_{1} x, x_{A}\right) \leq \varepsilon \text { for } i=N, \ldots, n .
$$

As a matter of fact, it turns out that the stability property established in this theorem is generic. That is, it holds for most (in the sense of Baire category) operators in the closure of $\mathcal{A}_{\text {reg }}$.

Theorem 1.4. The set $\mathcal{A}_{\text {reg }}$ contains an everywhere dense $G_{\delta}$ subset of $\overline{\mathcal{A}}_{\text {reg }}$.

The proofs of Theorems 1.1, 1.2, 1.3 and 1.4 are given in Sections 2, 3, 4 and 5 , respectively.

\section{Proofs of Theorem 1.1 And Corollary 1.1}

We first prove Theorem 1.1. To this end, set, for $x \in X$ and $r>0$,

$$
B(x, r)=\{y \in X: \rho(x, y) \leq r\} .
$$

We may assume without loss of generality that

$$
\varepsilon \leq 1 \text { and } B\left(x_{A}, 4\right) \subset K .
$$

By (A2), there exists a natural number $N \geq 4$ such that

$$
\rho\left(A^{n} x, x_{A}\right) \leq \varepsilon / 4 \text { for all integers } n \geq N \text { and all } x \in K \text {. }
$$

By (A4), the set $A^{m}(K)$ is bounded for all natural numbers $m$. Hence there exists a positive number $S>0$ such that

$$
A^{i}(K) \subset B\left(x_{A}, S\right), i=0, \ldots, 2 N .
$$


(Here we use the convention that $A^{0}$ is the identity operator.) By induction and (A3), we define a finite sequence of positive numbers $\left\{\gamma_{i}\right\}_{i=0}^{2 N}$ so that

$$
\gamma_{2 N}=\varepsilon / 4
$$

and, for each $i=0,1, \ldots, 2 N-1$,

$$
\gamma_{i} \leq \gamma_{i+1}
$$

and

$$
\rho(A x, A y) \leq 2^{-1} \gamma_{i+1} \text { for all } x, y \in B\left(x_{A}, S+4\right) \text { with } \rho(x, y) \leq \gamma_{i} .
$$

Set

$$
\delta=\gamma_{0} / 2
$$

Next, we prove the following auxiliary result.

Lemma 2.1. Suppose that $\left\{z_{i}\right\}_{i=0}^{2 N} \subset X$ satisfies

$$
z_{0} \in K \text { and } \rho\left(z_{i+1}, A z_{i}\right) \leq \delta, i=0, \ldots, 2 N-1 \text {. }
$$

Then

$$
\rho\left(z_{i}, x_{A}\right) \leq \varepsilon, i=N, \ldots, 2 N \text {. }
$$

Proof. We will show that for $i=1, \ldots, 2 N$,

$$
\rho\left(z_{i}, A^{i} z_{0}\right) \leq \gamma_{i} \text {. }
$$

Clearly, (2.8) holds for $i=1$ by (2.7) and (2.6).

Assume that $i \in\{2, \ldots, 2 N\}$ and

$$
\rho\left(z_{i-1}, A^{i-1} z_{0}\right) \leq \gamma_{i-1} \text {. }
$$

Then (2.7) implies that

$$
\begin{aligned}
\rho\left(z_{i}, A^{i} z_{0}\right) & \leq \rho\left(z_{i}, A z_{i-1}\right)+\rho\left(A z_{i-1}, A\left(A^{i-1} z_{0}\right)\right) \\
& \leq \delta+\rho\left(A z_{i-1}, A\left(A^{i-1} z_{0}\right)\right) .
\end{aligned}
$$

It follows from the definition of $\gamma_{i-1}$ (see (2.4)), (2.9), (2.7) and (2.3) that

$$
A^{i-1} z_{0}, z_{i-1} \in B\left(x_{A}, S+1\right) .
$$

By these inclusions, the definition of $\gamma_{i-1}$ (see (2.5) with $j=i-1$ ) and $(2.9)$,

$$
\rho\left(A\left(A^{i-1} z_{0}\right), A z_{i-1}\right) \leq \gamma_{i} / 2 .
$$

When combined with (2.10) and (2.6), this inequality implies that

$$
\rho\left(z_{i}, A^{i} z_{0}\right) \leq \delta+\gamma_{i} / 2 \leq \gamma_{i} .
$$


Therefore $(2.8)$ is valid for all $i \in\{1, \ldots, 2 N\}$. Together with (2.2), (2.4), (2.7) and (2.8), this last inequality implies that for all $i \in\{N, \ldots, 2 N\}$, we have

$$
\rho\left(z_{i}, x_{A}\right) \leq \rho\left(z_{i}, A^{i} z_{0}\right)+\rho\left(A^{i} z_{0}, x_{A}\right) \leq \gamma_{i}+\varepsilon / 4 \leq \varepsilon / 2 .
$$

Lemma 2.1 is proved.

Now we are ready to complete the proof of Theorem 1.1.

To this end, assume that $n \geq N$ is a natural number and that the sequence $\left\{x_{i}\right\}_{i=0}^{n} \subset X$ satisfies

$$
x_{0} \in K \text { and } \rho\left(A x_{i}, x_{i+1}\right) \leq \delta, i=0, \ldots, n-1 .
$$

We will show that

$$
\rho\left(x_{i}, x_{A}\right) \leq \varepsilon, i=N, \ldots, n .
$$

If $n \leq 2 N$, then (2.11) follows from Lemma 2.1. Therefore we may confine our attention to the case where $n>2 N$. Again by Lemma 2.1,

$$
\rho\left(x_{i}, x_{A}\right) \leq \varepsilon, i=N, \ldots, 2 N .
$$

Assume by way of contradiction that there exists an integer $q \in(2 N, n]$ such that

$$
\rho\left(x_{q}, x_{A}\right)>\varepsilon .
$$

In view of (2.12), we may assume without loss of generality that

$$
\rho\left(x_{i}, x_{A}\right) \leq \varepsilon, i \in\{2 N, \ldots, q-1\} .
$$

Define $\left\{z_{i}\right\}_{i=0}^{2 N} \subset X$ by

$$
z_{i}=x_{i+q-N}, i=0, \ldots, N, z_{i+1}=A z_{i}, i=N, \ldots, 2 N-1 .
$$

We will show that the sequence $\left\{z_{i}\right\}_{i=0}^{2 N}$ satisfies (2.7). To meet this goal, we only need to show that $z_{0} \in K$. By (2.15), (2.14) and (2.12),

$$
z_{0}=x_{q-N} \text { and } \rho\left(z_{0}, x_{A}\right) \leq \varepsilon .
$$

The last inequality and (2.1) imply that $z_{0} \in K$. Therefore (2.7) holds. It now follows from Lemma 2.1 and (2.15) that

$$
\rho\left(x_{A}, x_{q}\right)=\rho\left(x_{A}, z_{N}\right) \leq \varepsilon .
$$

This, however, contradicts (2.13). The contradiction we have reached proves (2.11) and this completes the proof of Theorem 1.1.

Finally, we are going to prove Corollary 1.1.

Set $K=\left\{x_{n}: n=0,1, \ldots\right\}$ and let $\varepsilon>0$ be given. Let $\delta>0$ and a natural number $N$ be as guaranteed by Theorem 1.1. There exists a natural number $j$ such that for each integer $i \geq j$, we have $\rho\left(A x_{i}, x_{i+1}\right) \leq \delta$. It follows from the last inequality and the choice of $\delta$ that $\rho\left(x_{i}, x_{A}\right) \leq \varepsilon$ for 
all integers $i \geq j+N$. Since $\varepsilon$ is an arbitrary positive number, this implies that $\lim _{i \rightarrow \infty} x_{i}=x_{A}$. The proof of Corollary 1.1 is complete.

\section{Proof of Theorem 1.2}

We may assume without loss of generality that $\varepsilon \leq 1$. By Theorem 1.1, there exist a natural number $N$ and $\delta_{0} \in(0, \varepsilon)$ such that the following property holds:

(P1) For each natural number $n \geq N$ and each sequence $\left\{y_{i}\right\}_{i=0}^{n} \subset X$ which satisfies

$$
y_{0} \in B\left(x_{A}, 4\right) \text { and } \rho\left(y_{i+1}, A y_{i}\right) \leq \delta_{0}, i=0, \ldots, n-1,
$$

the following inequality is true:

$$
\rho\left(y_{i}, x_{A}\right) \leq \varepsilon, i=N, \ldots, n .
$$

By property (A4), the set $A^{i}\left(B\left(x_{A}, 4\right)\right)$ is bounded for any integer $i \geq 1$. Choose a number $s>1$ such that

$$
\bigcup_{i=0}^{N} A^{i}\left(B\left(x_{A}, 4\right)\right) \subset B\left(x_{A}, s\right) .
$$

By induction and (A3), we define a finite sequence of positive numbers $\left\{\gamma_{i}\right\}_{i=0}^{N}$ so that

$$
\gamma_{i} \leq 1, i=0, \ldots, N, \gamma_{N} \leq \delta_{0} / 4, \gamma_{i} \leq \gamma_{i+1}, i=0, \ldots, N-1,
$$

and for each $j \in\{0, \ldots, N-1\}$,

$$
\rho(A x, A y) \leq 2^{-1} \gamma_{j+1} \text { for all } x, y \in B\left(x_{A}, s+4\right) \text { with } \rho(x, y) \leq \gamma_{j} .
$$

Set

$$
\delta=\gamma_{0} / 4
$$

Assume that $\left\{x_{i}\right\}_{i=0}^{\infty} \subset X$,

$$
\rho\left(x_{0}, x_{A}\right) \leq \delta \text { and } \rho\left(x_{i+1}, A x_{i}\right) \leq \delta, i=0,1, \ldots
$$

We will show that

$$
\rho\left(x_{i}, x_{A}\right) \leq \varepsilon
$$

for all integers $i \geq 0$. By (3.7), (3.6) and (P1), inequality (3.8) holds for all integers $i \geq N$. Therefore we only need to prove (3.8) for $i<N$. Clearly, (3.8) holds for $i=0$.

We will show that for $i=0, \ldots, N$, we have

$$
\rho\left(x_{i}, x_{A}\right)=\rho\left(x_{i}, A^{i} x_{A}\right) \leq \gamma_{i} .
$$


By (3.7) and (3.6), this is true for $i=0$. Assume that $i \in\{1, \ldots, N\}$ and

$$
\rho\left(x_{i-1}, A^{i-1} x_{A}\right)=\rho\left(x_{i-1}, x_{A}\right) \leq \gamma_{i-1} .
$$

Then (3.7) implies that

$$
\rho\left(x_{i}, x_{A}\right) \leq \rho\left(x_{i}, A x_{i-1}\right)+\rho\left(A x_{i-1}, x_{A}\right) \leq \delta+\rho\left(A x_{i-1}, x_{A}\right) .
$$

It follows from (3.10) and (3.4) that

$$
x_{i-1} \in B\left(x_{A}, s\right) .
$$

By (3.12), (3.10) and the definition of $\gamma_{i-1}$ (see (3.5) with $j=i-1$ ),

$$
\rho\left(A x_{i-1}, x_{A}\right) \leq 2^{-1} \gamma_{i} .
$$

Using (3.11), (3.13), (3.6) and (3.4), we obtain

$$
\rho\left(x_{i}, x_{A}\right) \leq \delta+2^{-1} \gamma_{i} \leq \gamma_{i} .
$$

Thus (3.9) indeed holds for all $i \in\{0, \ldots, N\}$. This fact, when combined with (3.4), implies that (3.8) is true for all $i \in\{0, \ldots, N\}$. This completes the proof of Theorem 1.2.

\section{Proof of Theorem 1.3}

We may assume, without loss of generality, that $\varepsilon<1$ and that $m \geq 1$ is a number such that

$$
m \geq \rho\left(x_{A}, \theta\right)+4 .
$$

By Theorem 1.1, there exist $\delta \in(0, \varepsilon)$ and a natural number $N$ such that the following property holds:

(P2) For each natural number $n \geq N$ and each sequence $\left\{x_{i}\right\}_{i=0}^{n} \subset X$ which satisfies

$$
x_{0} \in B(\theta, m) \text { and } \rho\left(A x_{i}, x_{i+1}\right) \leq \delta, i=0, \ldots, n-1,
$$

the following inequality holds:

$$
\rho\left(x_{i}, x_{A}\right) \leq \varepsilon, i=N, \ldots, n .
$$

Set

$$
K_{0}=B(\theta, m) \text { and } K_{i+1}=\left\{z \in X: \rho\left(z, A\left(K_{i}\right)\right) \leq 1\right\}, i=0,1, \ldots
$$

Clearly, the set $K_{i}$ is bounded for any integer $i \geq 0$. Choose a natural number $q \geq 8$ such that

$$
\bigcup_{i=0}^{2 N} K_{i} \subset B(\theta, q) \text { and } 1 / q<\delta / 8 .
$$

We are going to use the following technical result. 
Lemma 4.1. Assume that

$$
z \in B(\theta, m) \text { and }\left\{B_{i}\right\}_{i=1}^{2 N} \subset\left\{C \in \mathcal{A}:(C, A) \in E_{q}\right\},
$$

where $E_{q}$ is given by (1.1). Then

$$
\rho\left(B_{i} \ldots B_{1} z, x_{A}\right) \leq \varepsilon, i=N, \ldots, 2 N .
$$

Proof. Set

$$
z_{0}=z \text { and } z_{i}=B_{i} z_{i-1}, i=1, \ldots, 2 N .
$$

We will show that

$$
z_{i} \in K_{i}
$$

for $i=0, \ldots, 2 N$. Clearly, (4.9) holds for $i=0$. Assume that $i \in$ $\{0, \ldots, 2 N-1\}$ and (4.9) is valid. Inclusions (4.9) and (4.5) imply that

$$
z_{i} \in K_{i} \subset B(\theta, q) .
$$

When combined with (4.6), (1.1) and (4.8), this last inclusion implies that

$$
\rho\left(A z_{i}, z_{i+1}\right)=\rho\left(A z_{i}, B_{i+1} z_{i}\right) \leq 1 / q .
$$

Consequently, (4.11), (4.10) and (4.4) imply that $z_{i+1} \in K_{i+1}$. Therefore (4.9) is true for all $i=0, \ldots, 2 N$. This implies (see (4.5)) that

$$
\left\{z_{i}\right\}_{i=0}^{2 N} \subset B(\theta, q) .
$$

It follows from this inclusion, (4.5), (4.6) and (4.8) that for $i=0, \ldots, 2 N-1$,

$$
\rho\left(z_{i+1}, A z_{i}\right)=\rho\left(B_{i+1} z_{i}, A z_{i}\right) \leq 1 / q<\delta .
$$

By (P2), we see that

$$
\rho\left(B_{i} \ldots B_{1} z, x_{A}\right)=\rho\left(z_{i}, x_{A}\right) \leq \varepsilon, i=N, \ldots, 2 N .
$$

Lemma 4.1 is proved.

Now we are ready to complete the proof of Theorem 1.3. To this end, set

$$
\mathcal{U}=\left\{C \in \mathcal{A}:(C, A) \in E_{q}\right\} .
$$

Let $n \geq N$ be an integer, $x \in B(\theta, m)$, and $\left\{B_{i}\right\}_{i=1}^{n} \subset \mathcal{U}$. We will show that

$$
\rho\left(B_{i} \ldots B_{1} x, x_{A}\right) \leq \varepsilon \text { for } i=N, \ldots, n .
$$

If $n \leq 2 N$, then (4.13) follows from Lemma 4.1. Therefore we may restrict our attention to the case $n>2 N$. By Lemma 4.1,

$$
\rho\left(B_{i} \ldots B_{1} x, x_{A}\right) \leq \varepsilon, i=N, \ldots, 2 N .
$$

Suppose now that there exists an integer $p>2 N, p \leq n$, such that

$$
\rho\left(B_{p} \ldots B_{1} x, x_{A}\right)>\varepsilon .
$$


According to (4.14), we may assume, without loss of generality, that

$$
\rho\left(B_{i} \ldots B_{1} x, x_{A}\right) \leq \varepsilon, i=2 N, \ldots, p-1 .
$$

Define $\left\{D_{i}\right\}_{i=0}^{2 N} \subset \mathcal{A}$ by

$$
D_{i}=B_{i+p-N}, i=0, \ldots, N, D_{i}=A, i=N+1, \ldots, 2 N,
$$

and let

$$
z=B_{p-N} \ldots B_{1} x
$$

It follows from (4.14), (4.16), (4.17) and (4.1) that

$$
\rho\left(z, x_{A}\right) \leq \varepsilon \text { and } z \in B(\theta, m) .
$$

Applying now Lemma 4.1 to the mappings $\left\{D_{i}\right\}_{i=0}^{2 N}$ defined by (4.17), we deduce that

$$
\varepsilon \geq \rho\left(D_{N} \ldots D_{1} z, x_{A}\right)=\rho\left(x_{A}, B_{p} \ldots B_{p-N+1} z\right)=\rho\left(x_{A}, B_{p} \ldots B_{1} x\right),
$$

which contradicts (4.15). Hence (4.13) is true and Theorem 1.3 is established.

\section{Proof of Theorem 1.4}

Let $A \in \mathcal{A}_{\text {reg }}$ and let $k \geq 1$ be an integer. There is $x_{A} \in K$ such that

$$
A x_{A}=x_{A} .
$$

According to Theorem 1.3, there exist a natural number $N(A, k)$ and an open neighborhood $\mathcal{U}(A, k)$ of $A$ in $\mathcal{A}$ such that the following property holds:

(P3) For each $x \in B(\theta, k)$, each natural number $n \geq N(A, k)$ and each $B \in \mathcal{U}(A, k)$, we have $\rho\left(B^{n}, x_{A}\right) \leq 1 / k$.

Define

$$
\mathcal{F}=\left[\bigcap_{q=1}^{\infty} \bigcup\left\{\mathcal{U}(A, k): A \in \mathcal{A}_{\text {reg }} \text {, an integer } k \geq q\right\}\right] \cap \overline{\mathcal{A}}_{\text {reg }} .
$$

Clearly, $\mathcal{F}$ is an everywhere dense $G_{\delta}$ subset of $\overline{\mathcal{A}}_{\text {reg }}$.

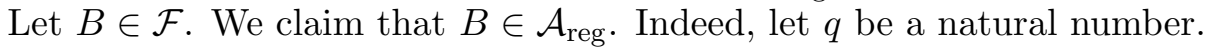
There exists a mappping $A_{q} \in \mathcal{A}_{\text {reg }}$ with a fixed point $x_{A_{q}}$ and a natural number $k_{q} \geq q$ such that

$$
B \in \mathcal{U}\left(A_{q}, k_{q}\right) .
$$

This inclusion together with (P3) imply that the following property holds:

(P4) For each $x \in B(\theta, q) \subset B\left(\theta, k_{q}\right)$ and each natural number $n \geq$ $N\left(A_{q}, k_{q}\right)$,

$$
\rho\left(B^{n} x, x_{A_{q}}\right) \leq k_{q}^{-1} \leq 1 / q
$$


Since $q$ is an arbitrary natural number, we obtain that for any $x \in X$, the sequence $\left\{B^{n} x\right\}_{n=1}^{\infty}$ is a Cauchy sequence and its limit is the unique fixed point $x_{B}$ of $B$. Thus

$$
\lim _{n \rightarrow \infty} B^{n} z=x_{B} \text { for any } z \in X .
$$

Property (P4) implies that

$$
\rho\left(x_{A_{q}}, x_{B}\right) \leq 1 / q
$$

Finally, it follows from property (P4) and (5.4) that for any $x \in B(\theta, q)$ and any $n \geq N\left(A_{q}, k_{q}\right)$,

$$
\rho\left(B^{n} x, x_{B}\right) \leq 2 / q .
$$

This implies that $B^{n} x \rightarrow x_{B}$ as $n \rightarrow \infty$, uniformly on any bounded subset of $X$. This completes the proof of Theorem 1.4.

\section{Note added in proof.}

(1) Corollary 1.1 provides a partial answer to an open question raised in [6] in the wake of Theorem 1 of [5], which is also concerned with the stability of iterations.

(2) Inexact orbits of nonexpansive mappings have recently been studied in [3]. We take this opportunity to correct a misprint in that paper: on page 28 , lines 10,11 and $17, x^{k+1}$ should be replaced with $x^{k+i}$. The inequality on line 17 holds then for all $i \geq i_{0}$.

Acknowledgments. A part of the first author's work on this paper was carried out when he was visiting the Institute of Mathematics of the Technical University of Łódź. The second author was partially supported by the Fund for the Promotion of Research at the Technion and by the Technion VPR Fund - B. and G. Greenberg Research Fund (Ottawa). All three authors are grateful to a referee for a careful reading of the manuscript and for many helpful comments.

\section{References}

[1] Browder, F. E., On the convergence of successive approximations for nonlinear functional equations, Indag. Math. (N.S.) 30 (1968), 27-35.

[2] Butnariu, D., Reich, S., Zaslavski, A. J., Asymptotic behavior of relatively nonexpansive operators in Banach spaces, J. Appl. Anal. 7 (2001), 151-174.

[3] Butnariu, D., Reich, S., Zaslavski, A. J., Convergence of fixed points of inexact orbits of Bregman-monotone and of nonexpansive operators in Banach spaces, in "Fixed Point Theory and its Applications", Yokohama Publishers, Yokohama, 2006, 11-32.

[4] De Blasi, F. S., Myjak, J., Sur la porosité de l'ensemble des contractions sans point fixe, C. R. Acad. Sci. Paris Sér. I Math. 308 (1989), 51-54. 
[5] Jachymski, J., An extension of A. Ostrowski's theorem on the round-off stability of iterations, Aequationes Math. 53 (1997), 242-253.

[6] Jachymski, J., Józwik, I., Nonlinear contractive conditions: a comparison and related problems, Banach Center Publ. 77 (2007), 123-146.

[7] Krasnosel'skii, M. A., Zabreiko, P. P., Geometrical Methods of Nonlinear Analysis, Springer, Berlin, 1984.

[8] Ostrowski, A. M., The round-off stability of iterations, ZAMM Z. Angew. Math. Mech. 47 (1967), 77-81.

[9] Rakotch, E., A note on contractive mappings, Proc. Amer. Math. Soc. 13 (1962), 459-465.

[10] Reich, S., Shafrir, I., Nonexpansive iterations in hyperbolic spaces, Nonlinear Anal. 15 (1990), 537-558.

[11] Reich, S., Zaslavski, A. J., Convergence of generic infinite products of affine operators, Abstr. Appl. Anal. 4 (1999), 1-19.

[12] Reich, S., Zaslavski, A. J., Convergence of generic infinite products of orderpreserving mappings, Positivity 3 (1999), 1-21.

[13] Reich, S., Zaslavski, A. J., The set of noncontractive mappings is $\sigma$-porous in the space of all nonexpansive mappings, C. R. Acad. Sci. Paris Sér. I Math. 333 (2001), 539-544.

DAN BUTNARIU

DEPARTMENT OF MATHEMATiCS

UNIVERSITY OF HAIFA

31905 HAIFA

ISRAEL

E-MAIL:DBUTNARU@MATH.HAIFA.AC.IL
SimeON REICH

DEPARTMENT OF MATHEMATICS

THE TECHNION-ISRAEL

Institute OF TECHNOLOGY 32000 HAIFA, ISRAEL

AlEXANDER J. ZASLAVSKI

DEPARTMENT OF MATHEMATICS

The Technion-Israel Institute of Technology

32000 HAIFA, ISRAEL

E-MAIL: AJZASL@TX.TECHNION.AC.IL 